\title{
La identificación de los grupos de interés de las entidades sin fines de lucro en la emisión de información transparente
}

\author{
Miren Lorea Maguregui Urionabarrenechea ${ }^{1}$, Javier Corral Lage ${ }^{2}$ y Crisanta Elechiguerra \\ Arrizabalaga $^{3}$
}

Recibido: 21 de octubre de 2018 / Aceptado: 27 de noviembre de 2018

Resumen. Actualmente, la sociedad se está concienciando de que cualquier tipo de organización debe informar y comunicar de manera veraz, clara y objetiva. Este proceso está relacionado con su nivel de transparencia. Precisamente, los diferentes grupos de interés o stakeholders de las Entidades Sin Fines de Lucro (ESFL) han incrementado de manera notable su demanda de información con una notificación más amplia de sus actividades. Con la entrada en vigor de la Ley 19/2013, de transparencia, acceso a la información pública y buen gobierno, se normativiza el proceso. Sin embargo, la obligación de publicidad activa que plantea es necesaria pero no suficiente, si tenemos en cuenta también las propias características y vicisitudes del Sector.

Así, el objetivo del trabajo es identificar, desde el punto de vista de las propias ESFL, quiénes pueden ser considerados como sus grupos de interés y deben demandar una información transparente. Asimismo, se pretende determinar cuáles son los atributos que la emisión de una información transparente aporta a estas entidades. La metodología empleada ha sido fundamentalmente una exhaustiva revisión bibliográfica, complementada con un estudio empírico a través de un cuestionario en el que se recoge la opinión de 140 ESFL españolas. Los resultados obtenidos, después de analizados, han permitido elaborar unas conclusiones relevantes. Se destaca la necesidad cada vez mayor de impulsar la transparencia como mecanismo de supervivencia y calidad de las ESFL, y de concienciar que comunicar una información transparente no es una carga adicional en la labor que desempeñan, sino una posibilidad para ofrecer un valor añadido a la organización.

Palabras clave: Transparencia; ONG; Usuarios; Información; Sociedad.

Claves Econlit: A13; L31; M41.

\section{[en] Identifying stakeholders of non-profit organizations in the issuance of transparent information}

Abstract. Currently, society is becoming aware that any organization must inform and communicate accurately, clearly and objectively. This process is related to its level of transparency. Thus, the different stakeholders of non-profit entities have increased significantly their demand for information with a broader notification of their activities. The process is regulated with the entry into force of Law 19/2013, of transparency, access to public information and good governance. However, if we consider

1 Universidad del País Vasco, España

Dirección de correo electrónico: lorea.maguregi@ehu.eus

2 Universidad del País Vasco, España

Dirección de correo electrónico: javier.corral@ehu.eus

3 Universidad del País Vasco, España

Dirección de correo electrónico: crisanta.elechiguerra@ehu.eus

Revesco (131) 2019: 65-85 
the characteristics and vicissitudes of the sector, its obligation of active advertising is necessary but not sufficient.

Thus, the study aims to identify, from the point of view of the nonprofit organizations, who can be considered as their stakeholders and should demand transparent information. Likewise, the aim is to determine the attributes that the issuance of transparent information brings to these entities. The methodology used has been an exhaustive bibliographic review, complemented by an empirical study through a questionnaire in which the opinion of 140 Spanish nonprofit organizations is collected. The results obtained after being analyzed have allowed the development of some relevant conclusions. We stand out the growing need to promote transparency as a mechanism of survival and quality of NGO and to aware that the communication of transparent information is not an additional burden on the work performed, but the possibility of providing added value to the organization.

Keywords: Transparency; NGO; Users; Information; Society.

Sumario. 1. Introducción. 2. La necesidad de emisión de información transparente en las entidades sin fines de lucro. 3. Identificación de los grupos de interés de las entidades sin fines de lucro. 4. Metodología. 5. Análisis y resultados. 6. Conclusiones. 7. Futuras líneas de investigación. 8. Referencias bibliográficas.

Cómo citar: Maguregui Urionabarrenechea, M.L., Corral Lage, J. y Elechiguerra Arrizabalaga, C. (2019) La identificación de los grupos de interés de las entidades sin fines de lucro en la emisión de información transparente. REVESCO. Revista de Estudios Cooperativos, Segundo Cuatrimestre, N $^{\circ}$ 131, pp. 65-85. DOI: 10.5209/REVE.62814.

\section{Introducción}

Las entidades sin fines de lucro (ESFL) son entes privados, organizados formalmente, con autonomía de decisión, de libre adhesión, con importante componente voluntario, cuyo objetivo es fundamentalmente el interés general y cuyos excedentes, de haberlos, revierten en ayudar a la entidad a cumplir con su misión (Salamon y Sokolowski, 2016). En ellas, a diferencia de las organizaciones lucrativas, la toma de decisiones no depende de si se tiene parte o no en la financiación (García-Gutiérrez, 2002). Además, atendiendo a los criterios de participación, las ESFL poseen un marcado grado corporativo respecto al operativo. Mientras que la participación operativa ha sido, en principio, considerada como fuente de competitividad en las organizaciones lucrativas (Pérez, 2002), la participación corporativa deriva una serie de ventajas en lo que a eficiencia se refiere, vislumbradas por el consentimiento de la perspectiva stakeholder.

Asimismo, en los últimos años, la sociedad se está concienciando de la necesidad de que cualquier tipo de organización debe informar de manera veraz, clara y objetiva (Goodpaster, 1991). Este proceso de sensibilización está directamente relacionado con el nivel de transparencia de las entidades, en la medida en que éstas ofrezcan información amplia, ágil y rigurosa de sus actividades y objetivos de cara a los intereses sociales comunes (Âlvarez, 2010). Por ello, actualmente, los diferentes stakeholders de las ESFL han incrementado de manera exponencial su demanda de información exigiendo una notificación aún más amplia de sus actividades (Moneva y Bellostas, 2007; Marcuello, Bellostas, Marcuello y Moneva, 2007). Por lo tanto, la generación de un clima de confianza y credibilidad entre las ESFL y sus grupos de interés ayudará a legitimar sus 
actuaciones, siendo la transparencia un elemento crucial para ello (Balas-Lara, 2011).

Ante la entrada en vigor de la Ley 19/2013, de 9 de diciembre, de transparencia, acceso a la información pública y buen gobierno (Ley de Transparencia), se normativiza la obligación de las ESFL de informar de manera transparente a sus usuarios de información. Sin embargo, la obligación de publicidad activa de la información que plantea la Ley y su proyecto de Reglamento para aquellas ESFL privadas sujetas a la misma, es necesaria pero no suficiente si tenemos en cuenta la demanda de sus grupos de interés y las propias características y vicisitudes del Sector No Lucrativo. Por lo que es fundamental ofrecer un mayor grado de información a la sociedad en general.

El objetivo del trabajo es concienciar de la necesidad de educar y formar a las ESFL en comunicar transparentemente. Para ello, a través de un estudio empírico, se identifica quiénes son sus principales grupos de interés y quiénes pueden demandar una información transparente. Asimismo, se comprueba qué aspectos favorables ofrece ésta a las ESFL y se examina cuáles son las características que debe cumplir.

La metodología empleada ha sido fundamentalmente una exhaustiva revisión bibliográfica, la cual ha sido, como hemos reseñado, complementada a su vez con un estudio empírico cuyos resultados, después de analizados, han permitido elaborar unas conclusiones relevantes.

\section{La necesidad de emisión de información transparente en las entidades sin fines de lucro}

\subsection{La transparencia en la emisión de información externa en las ESFL}

En la actualidad, se está otorgando un elevado nivel de importancia a la transparencia en el Sector No Lucrativo, de acuerdo con los estudios realizados por Kearns (1994), el Observatorio del Tercer Sector (2007), Vidal et al. (2008) y Cavanna (2013), entre otros.

Las definiciones del concepto de transparencia pueden ser numerosas en función de los autores que se han interesado por dicha acepción (Gruber, 1987; Shafritz, 1992; Puig, 1999; Kearns, 2001; Bonbright, 2007; Vidal et al., 2008; Vaccaro y Madsen, 2009; Starling, 2010; entre otros). Sin embargo, tras ellos se aprecia una posición en común entre unos y otros. En general, se entiende la transparencia como aquella cualidad que permite a todos los grupos de interés conocer y descubrir el funcionamiento y la identidad de una organización (Herranz de la Casa, 2006).

Una buena comunicación tiene el influjo de crear una imagen atractiva de una organización y una posición sólida de diferenciación frente al resto (Salvador I Peris, 2003). A estos hechos debemos unirles que el rol de transformación social que poseen las ESFL establece, además, un compromiso que implica una exigencia social, lo que plantea el reto de implicar e involucrar a la sociedad en las tareas que realizan y en los objetivos que persiguen. En este marco se hace necesario impulsar la transparencia para con todos aquellos que formen parte del proceso (Vidal et al., 
2008) y de esta manera comunicar a los grupos de interés una información veraz (Balas-Lara, 2008), objetiva (Zhang, Griffith, Pershing, Sun, Malakoff, Marsland y Field, 2017), clara (Saz-Gil, 2008), concisa (Balser y McClusky, 2005; Waters, Burnett, Lamm, y Lucas, 2009), útil (Bernal, 2013) y fiable y completa (Val, Bernat, y Johanisova, 2012).

Asimismo, la comunicación en la organización es un atributo imprescindible para transmitir una visión determinada de ésta, para informar sobre su misión, objetivos y actividades a desarrollar. Relacionarse con otros agentes sociales de un modo transparente, respetuoso y coherente con los valores propios de las ESFL se hace fundamental a la hora de desarrollar cauces de comunicación entre las diferentes partes interesadas (Vidal et al., 2008).

Por lo tanto, impulsar la transparencia como mecanismo de supervivencia y garantía de calidad de las ESFL resulta cada vez más necesaria para comunicar una imagen más positiva del Sector (Mitchell, Agle y Wood 1997). Así, se debe tener claro que la confianza en estas entidades es extremadamente frágil, puesto que es muy difícil conseguirla, pero muy fácil perderla. Consecuentemente, el principal desafío de una ESFL es obtener legitimidad de cara a todos aquellos que de una manera $u$ otra se relacionan con ella, siendo por lo tanto clave la transparencia como elemento fundamental para garantizar dicha legitimidad (Herlin, 2015), así como confianza (Herman y Renz, 1999; Fisher, 2015), credibilidad social (Brown y Moore, 2001; Huang, 2017), honestidad (Rhine, 2015) y legalidad (Elechiguerra, Corral-Lage y Maguregui, 2015; Peng, Pandey y Pandey, 2015) para que éstas puedan desarrollarse y avanzar en el cumplimiento de su misión.

\subsection{La Ley de Transparencia}

Con la llegada de la Ley de Transparencia se da un paso adelante en la búsqueda de una emisión de información más clara, completa, homogénea y sobre todo transparente hacia los grupos de interés de las ESFL privadas. Nace como un paso fundamental y necesario ante la insuficiencia que existía de una regulación explícita. Pero no sólo eso, los casos de fraude y corrupción que empañaron y empañan la sociedad actual derivó en una demanda de una mejora en este tema.

El objeto del Texto Consolidado es ampliar y reforzar la transparencia de la actividad pública, regular y garantizar el derecho de acceso a la información y establecer las obligaciones que deben cumplir los responsables públicos sobre buen gobierno, así como las consecuencias derivadas de su incumplimiento. Se concluye que el ámbito de actuación es eminentemente de carácter público pudiendo darse la idea de que nos encontramos ante una Ley que deja de lado al conjunto de asociaciones y fundaciones privadas.

Sin embargo, de acuerdo con el artículo tercero, sobre "Otros sujetos obligados" del Capítulo I denominado ámbito subjetivo de aplicación del Título I sobre transparencia de la actividad pública, en su segundo párrafo nos indica que las disposiciones del Capítulo II relativas a la publicidad activa, afectan en materia de información de carácter institucional, organizativa y de planificación y en materia económica a ciertas ESFL privadas. Así, serán sujetos obligados de esta Ley las siguientes entidades: a) Los partidos políticos, organizaciones sindicales y organizaciones empresariales; b) Las entidades privadas que perciban durante el 
período de un año ayudas o subvenciones públicas en una cuantía superior a 100.000 euros o cuando al menos el $40 \%$ del total de sus ingresos anuales tengan carácter de ayuda o subvención pública, siempre que alcancen como mínimo la cantidad de 5.000 euros. Si nos fijamos en el apartado b) podemos interpretar que son varias las ESFL que pueden verse obligadas a llevar a cabo una publicidad activa de la información que requiera la Ley, siempre y cuando cumpla con los requisitos del ámbito de aplicación. Ahora bien, en el apartado b) sólo explicita a aquellas organizaciones que reciban financiación pública y cumplan con los límites establecidos en el artículo 3, dejando fuera de los criterios la financiación de carácter privado.

No obstante, es una publicidad de mínimos, por lo que gran parte de la información que demandan sus grupos de interés queda relegada a la voluntariedad. Por lo que, mientras se desarrolla nueva normativa sustantiva en este aspecto, las ESFL poseen mecanismos voluntarios como memorias de sostenibilidad o indicadores socio-económicos, entre otros.

\subsection{Educar y formar a las ESFL en materia de información transparente}

Comunicar una información transparente no debe ser tomada como una carga adicional en la labor que desempeña una ESFL, sino como la posibilidad de ofrecer un valor añadido a la organización. La sociedad, y todos los grupos de interés que la conforman, demandan cada vez una información más completa y transparente. Dicha comunicación redunda de manera positiva en las propias entidades generándoles, como hemos comentado, una imagen positiva, una legitimidad y una credibilidad, entre otras. Por lo que las organizaciones del Sector No Lucrativo no han de ser reacias al cambio.

Se necesitan sistemas de educación dirigidos a las ESFL mediante encuentros, cursos o talleres que impliquen no sólo a las organizaciones, sino también a las administraciones públicas e investigadores del ámbito social porque sus recursos, tanto humanos como materiales, son escasos. Es fundamental explicarles qué deben hacer, cómo deben desarrollar y para qué deben llevar a cabo procesos de emisión de información transparente, así como enseñarles cuáles son las herramientas que pueden utilizar y cómo interpretarlas.

Asimismo, el proceso de educación y formación mediante dinámicas participativas donde se analiza la situación real de las ESFL mediante un diagnóstico y se plantea cómo mejorarla, no ha de ser unidireccional, sino que se puede y se debe implicar a las entidades en el proceso, para generar así una retroalimentación que permita maximizar las sinergias.

\section{Identificación de los grupos de interés de las entidades sin fines de lucro}

\subsection{Los grupos de interés y la Teoría de los Stakeholders}

La evolución de la acepción stakeholders ha sufrido alteraciones a medida que las aplicaciones del término eran realizadas desde diferentes perspectivas, y es a partir de mediados de los ochenta cuando comienza a lograr relevancia y notoriedad. Han 
sido muchos los estudios relacionados desde la aportación de Freeman en 1984: Fottler (1987), Clarkson (1995), Donaldson y Preston (1995), Gray et al. (1997), Kaler (2002), Carrasco, Correa-Ruíz, y Larrinaga (1999), Larrinaga et al. (2002), Unerman y Bennet (2004), Escobar y González (2005), Unerman (2007), Bebbington, Larrinaga, y Moneva (2008), Álvarez-Etxeberria (2009), Horrach y Socías-Salvà (2011), entre otros.

En la actualidad, el concepto está adquiriendo mayor relevancia y cada vez son más los grupos que deben ser considerados como stakeholders. Han sido muchos los autores coetáneos a Freeman como Grunig y Hunt (1984), Thompson, Wartick y Smith (1992), Starik (1994), Steadman, Zimmerer y Green (1995), Gabas, Monerva, Bellostas y Jarne (1996), Johnson, Scholes y Fréry (2000), Kaler (2002), Caballero (2006), entre otros, cuyas definiciones, aun basándose en la de éste, han divergido en función de la amplitud tenida en cuenta. Así, el término stakeholder hace referencia a un individuo o ente con interés en la organización (Caballero, 2006) y con el que la entidad se encuentra interrelacionada de una manera u otra, puesto que verdaderamente éste es demandante de información. Es decir, es aquél o aquellos con derecho a preguntar o con motivos para hacerlo (Álvarez, 2010) y que a su vez influyen y son influidos por la organización (Gray et al., 1997), tanto de manera activa como pasiva, sin cuyo apoyo ésta dejaría de existir (Montero y Araque, 2006).

En definitiva, desde la visión de los stakeholders lo que se procura es proporcionar un estándar de gestión que sea expresión de la responsabilidad de carácter social de las ESFL. Por lo que su análisis resulta valioso a la hora de afrontar el estudio de las interrelaciones en una organización, para así, mejorar la gestión de la misma, principalmente en lo que a emisión de información transparente se refiere.

El modelo de entidad orientado a satisfacer los intereses únicos de los suministradores de capital financiero a través de la Teoría de los Shareholders (Friedman, 1962) ha quedado anquilosado. El modelo actual centrado en la Teoría Stakeholder (Freeman, 1984) sostiene que todos los partícipes, denominados grupos de interés, implantan una relación de intercambio, de tal modo que los intereses y los beneficios entre la organización y cada uno de ellos sean recíprocos. Sus manifestaciones se han ido perfeccionando y autores como Post, Preston y Sachs (2002) aportan argumentaciones sobre el flujo de los beneficios, las relaciones múltiples, los roles simultáneos y la variación en los asuntos. En la teoría se defiende que no existen preferencias preestablecidas en lo referente a la importancia de unos intereses o beneficios respecto a otros. En la práctica, sin embargo, distará en función del tipo de organización y la actividad que desarrolle.

\subsection{Los roles de los grupos de interés de las ESFL}

Partiendo de que toda persona física o jurídica puede ser un stakeholder, las ESFL deben centrar sus esfuerzos en identificarlos y clasificarlos. Aunque una única clasificación generalista, no del todo exhaustiva, puede incurrir en el peligro de excluir ciertos elementos potencialmente relevantes, un intento de agrupamiento ayudará a facilitar el análisis de las relaciones de la organización con su entorno (Elechiguerra, Corral-Lage y Maguregui, 2015). 
Para identificar y clasificar los stakeholders en una ESFL, inicialmente es necesario buscar las respuestas a las siguientes cuestiones:

- Quiénes pueden ser demandantes de información.

- Cuáles son los papeles o roles que pueden desempeñar.

- $\quad$ Qué tipo de stakeholder es cada agente.

El estudio e identificación de las relaciones existentes entre la ESFL y sus stakeholders adquiere una mayor entidad si tenemos en cuenta los distintos papeles que estos pueden llegar a desempeñar. Dicha labor se convierte en una ardua tarea debido a:

- La heterogeneidad de organizaciones que forman parte del Sector No Lucrativo. Hecho que conllevaría un estudio específico por cada entidad, centrándose en las peculiaridades de cada una.

- $\quad$ La complejidad de las relaciones que este tipo de entidades entabla con sus diferentes grupos de interés, ya que pueden dar lugar a una infinidad de correlaciones.

Inicialmente, partiendo de Montero y Araque (2006), se presenta el marco de relaciones en una ESFL, distinguiendo las que provienen de sus actividades de las que se derivan del entorno.

En lo referente a los stakeholders de la actividad, las ESFL realizan actividades diversas y dispares, por lo que resulta necesario distinguir los diferentes elementos de actividad que se relacionan con la entidad. Estos pueden concebirse como corrientes de entrada (inputs de actividad) o de salida (outputs de actividad) puesto que las relaciones serán recíprocas. Tanto los inputs como los outputs pueden ser materiales (bienes y servicios) y humanos (factor trabajo).

Ilustración. 1. Roles de los grupos de interés de las ESFL

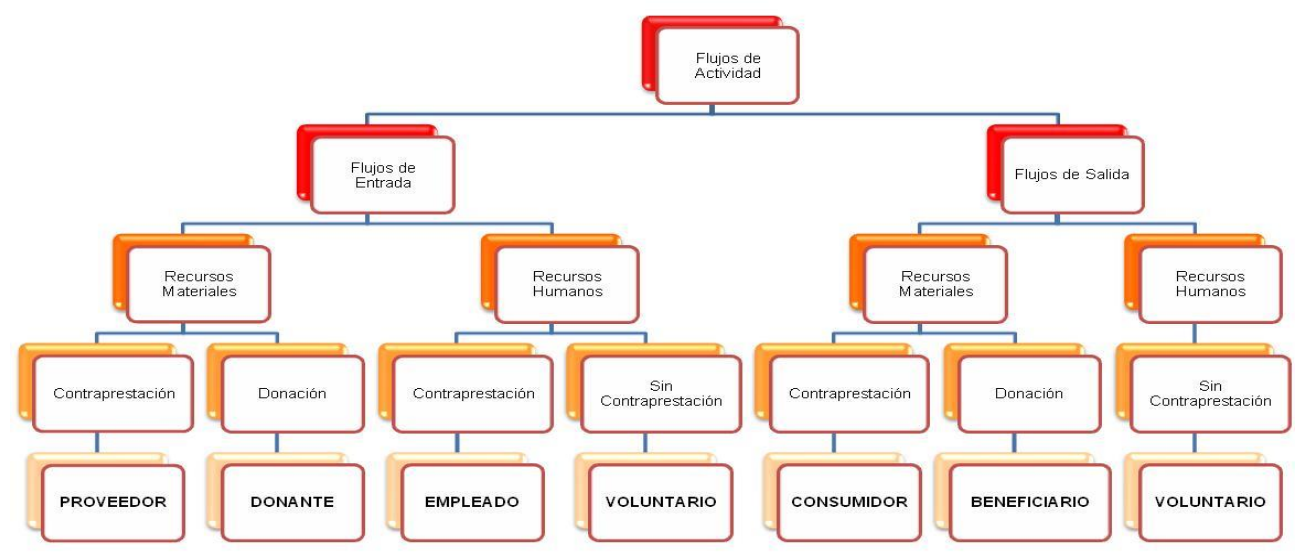

Fuente: elaboración propia a partir de Montero y Araque (2006) 
Además, los grupos de interés a identificar y clasificar diferirán en función de si existe o no una contraprestación de carácter económico. En el caso de los bienes y servicios que recibe, si estos son de una manera gratuita, nos encontramos ante una donación llevada a cabo por un donante (figura que engloba a los socios o patronos), mientras que, por el contrario, si dichos inputs han de ser abonados económicamente, nos referimos a los roles de acreedor.

En lo concerniente al trabajo, también se produce una dicotomía a causa de la retribución económica; cumplirá un papel de empleado aquel individuo que reciba un pago de un salario por parte de la organización, y de voluntario aquel que ejerza su labor sin recibir sueldo alguno.

Referente a los outputs de actividad, la direccionalidad es la inversa, es decir, el flujo va desde la entidad hacia el exterior. Además, los roles que ejercen los diferentes stakeholders difieren del caso anterior y distinguimos, únicamente, entre usuario y deudor (ver ilustración 1).

\subsection{Las correspondencias con el entorno de los grupos de interés de las ESFL}

Con respecto a los stakeholders relacionados con el entorno, debido al impacto social que promueven sus actividades, las ESFL son más abiertas al entorno, con un contacto mucho más directo con el exterior, lo que condiciona sus usuarios y necesidades. Por ello, es preciso agruparlos en función de los roles que puedan surgir. El hecho de que la dirección de una ESFL pretenda ir a la par con el entorno, acrecienta la importancia de la interacción entre ésta y sus stakeholders, por lo que se confiere como una tarea valiosa y necesaria.

Las relaciones pueden ser, inspirados en Montero y Araque (2006), recíprocas y no recíprocas. En estas últimas el objetivo principal de la ESFL es actuar sobre diferentes estamentos y sobre la sociedad en general para ejercer presión que modifique distintos comportamientos sociales. Nos encontramos ante un esfuerzo organizado, dirigido por un grupo o agente de cambio (en este caso la ESFL), que intenta persuadir a otros de que acepten, modifiquen o abandonen ciertas ideas, actitudes, prácticas y conductas.

En otras ocasiones, la presión es ejercida de manera opuesta, por el Sector Público, el Sector Capitalista, etc. Estos se convierten en críticos de las acciones de la ESFL, normalmente persiguiendo un beneficio particular. Nos encontramos ante los conocidos como grupos de presión, en el primer caso ejercido por la ESFL, y en el segundo hacia la entidad. Las relaciones recíprocas son aquéllas que se producen en ambas direcciones, y pueden diferir considerablemente, según se opte por una relación de competencia o de colaboración.

Dicha clasificación es fundamental para las ESFL, ya que conocer a sus grupos de interés les permite satisfacer sus necesidades de manera más eficiente, lo que redundará en un mayor apoyo social y una mejora de la sociedad en general (ver Ilustración 2). 
Ilustración. 2. Correspondencias con el entorno de los grupos de interés de las ESFL

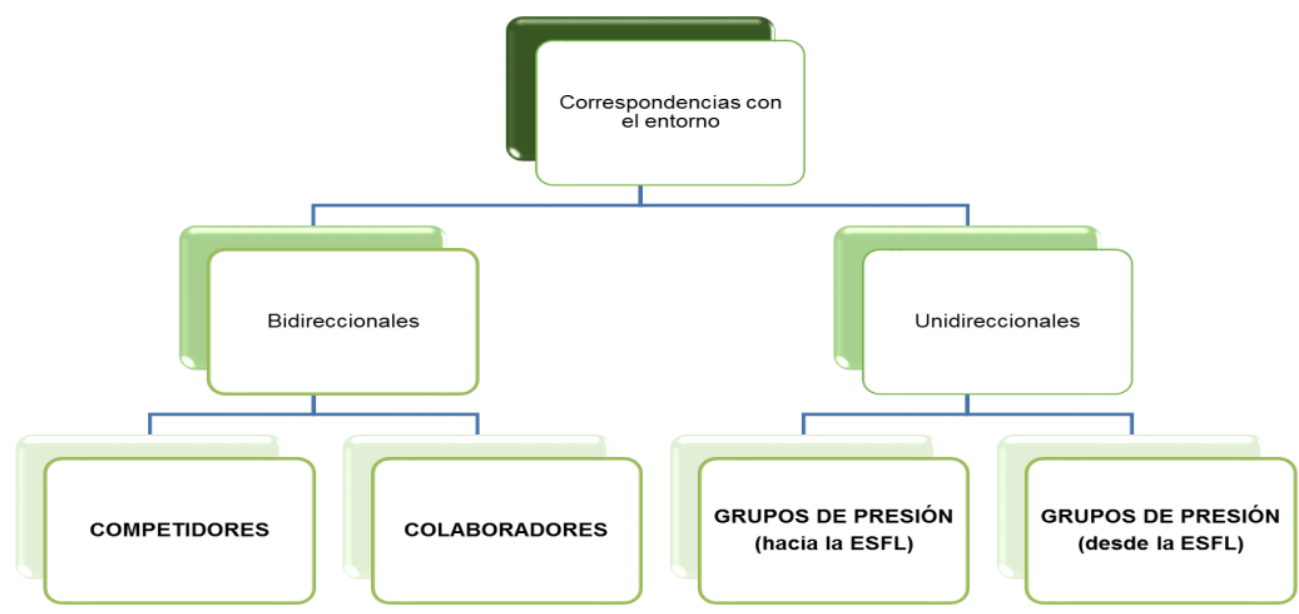

Fuente: elaboración propia a partir de Montero y Araque (2006)

\subsection{Clasificación de los grupos de interés de las ESFL atendiendo a los ámbitos de pertenencia}

Los stakeholders pueden llegar a adquirir diferentes roles que varían en consonancia con las circunstancias del contexto socio-económico de la ESFL. Por ello, definir quiénes son y qué papeles desempeñan, resulta una labor necesaria e imprescindible.

Se aprecia cómo no todos los stakeholders pueden pertenecer a todos los ámbitos, y cómo dentro de un estado económico concreto, varios son los partícipes que lo conforman. Así, tras la definición de quiénes son y qué papeles desempeñan los diferentes grupos de interés de una entidad no lucrativa, apreciamos la disparidad y número de agentes que actúan en el entorno más cercano de estas entidades.

También debemos tener en cuenta que dichos stakeholders, entendidos como agentes con entidad propia, pueden llegar a adquirir diferentes roles o éstos ir variando en consonancia con las circunstancias cambiantes del contexto socioeconómico.

Por ello, la tarea de definir quiénes son y que papeles desempeñan, aun siendo necesaria e imprescindible, resulta escasa para la misión de estudiar a los stakeholders como elementos creadores de ventaja competitiva para la organización, siendo necesario analizar qué tipo de información demandan (ver Ilustración 3). 
74 Maguregui Urionabarrenechea, M.L., Corral Lage, J. y Elechiguerra Arrizabalaga, C. Revesco (131) 2019: 65-85

Ilustración. 3. Relación de la clasificación de los grupos de interés de las ESFL de acuerdo con su ámbito de pertenencia

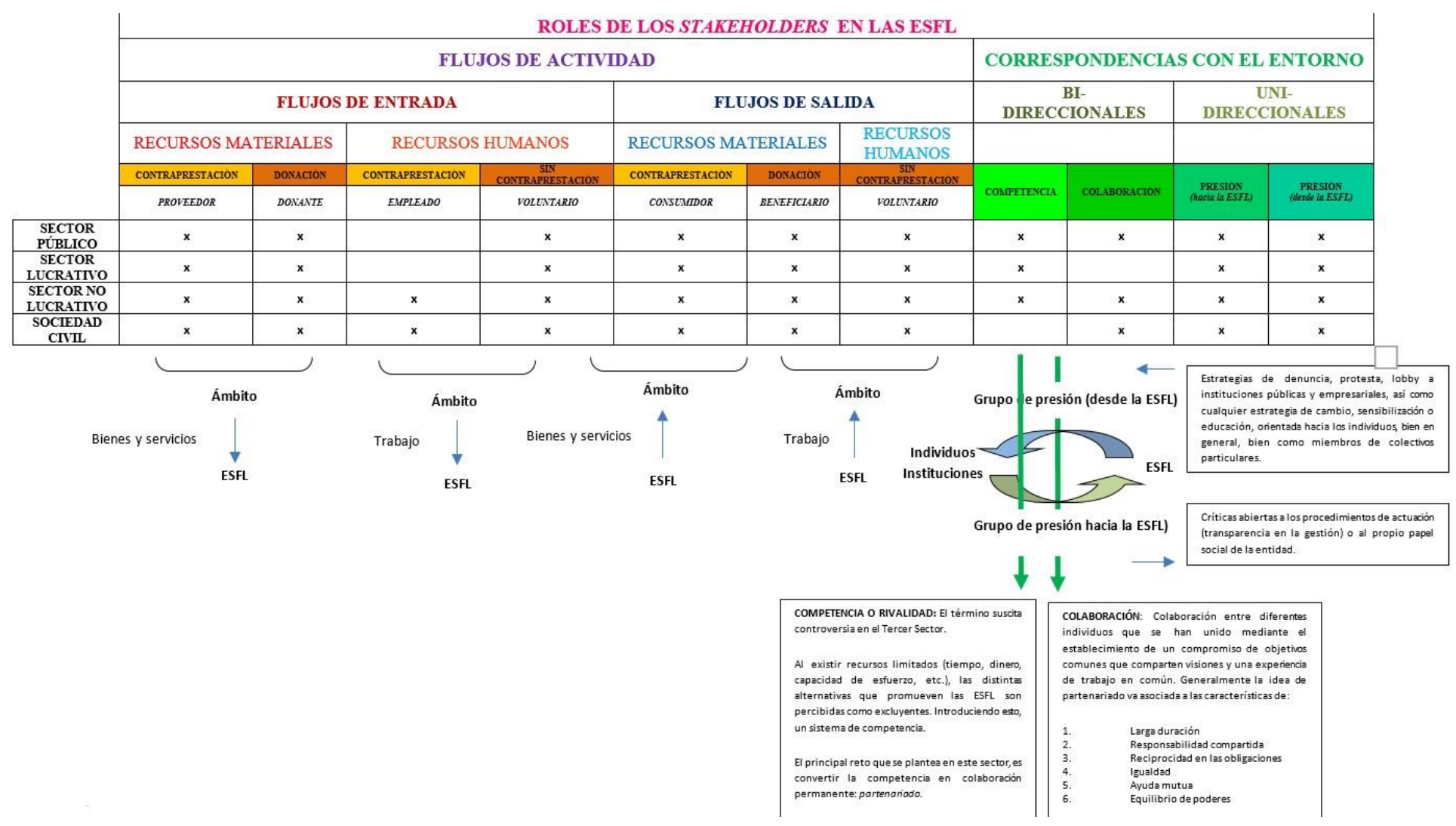

Fuente: elaboración propia 


\section{Metodología}

Tras estos planteamientos teóricos, se presenta el estudio que analiza, en el caso de las ESFL, la importancia de comunicar con transparencia a sus principales stakeholders. El trabajo realizado recurre a la herramienta metodológica y cuantitativa del cuestionario porque permite estudiar un fenómeno social como un proceso dinámico y dentro de su contexto real (Callejo, 2002).

Se ha pasado un cuestionario a aquellos responsables de la rendición de cuentas de diferentes ESFL a nivel estatal, en activo y que emitan información relevante para sus grupos de interés por medio de página web.

Los datos fueron tomados durante el ejercicio 2017 entre los responsables de emisión de información relevante para los stakeholders, de las ESFL encuestadas. Dichas organizaciones debían estar en activo durante al menos el año 2016 y haber utilizado hasta entonces su página web o similar como medio de comunicación de la información.

El tamaño muestral se ha determinado para lograr que el margen de error máximo sea inferior a 0,05 puntos, en este caso de $\pm 5 \%$ con un nivel de confianza del $95 \%(\mathrm{z}=1.96$, para $\mathrm{e}=0.05)$ sobre un Universo de 287.762 asociaciones y fundaciones, definido como finito.

$$
\mathrm{n}=\frac{\mathrm{N}}{1+\frac{\mathrm{e}^{2}(\mathrm{~N}-1)}{\mathrm{z}^{2} \mathrm{pq}}}
$$

Así, la muestra asciende a 384. Teniendo en cuenta las respuestas consideradas correctas, el número definitivo de encuestas asciende a 140. Esta cifra se considera representativa, puesto que suponen un índice de respuestas correctas del 36,45\% respecto al tamaño muestral.

Del total de encuestados, se recibió respuesta de 82 asociaciones $(58,57 \%)$ y 58 fundaciones $(41,43 \%)$. De todas ellas, 50 entidades $(35,71 \%)$ ejercen su actividad a nivel autonómico, provincial o municipal; 32 organizaciones $(22,86 \%)$ a nivel estatal; y las restantes 58 ESFL $(41,43 \%)$ a nivel internacional. En relación a las Comunidades Autónomas donde mayor nivel de respuesta se ha obtenido, encabeza la lista Madrid (61 entidades: 43,57\%); seguida muy de cerca por Cataluña (56 entidades: 40,00\%); Andalucía (47 entidades: 33,57\%); y Valencia y País Vasco (41 entidades: 29,29\%, ambas). Mayoritariamente nos encontramos con pequeñas y medianas entidades (69 organizaciones: 49,29\%), seguido de grandes entidades (45 organizaciones: $32,14 \%$ ) y finalmente microentidades (26 organizaciones: $18,57 \%$ )

\section{Análisis y resultados}

En el trabajo empírico se han obtenido los siguientes resultados: 


\subsection{Determinación de la importancia que supone para las ESFL comunicar con transparencia}

Tratando de profundizar en la transparencia y los factores que la rodean, se plantea a ambos colectivos cuál es el grado de relevancia que supone que las ESFL ofrezcan una información transparente a sus grupos de interés.

La inmensa mayoría de las ESFL encuestadas considera muy importante comunicar con transparencia. Aspecto éste que nos obliga, tras identificar y clasificar sus grupos de interés, a comprobar cuáles deben ser considerados como principales usuarios de la información que emiten estas entidades (ver Ilustración $4)$.

Ilustración. 4. ¿Qué nivel de importancia supone comunicar con transparencia por parte de las ESFL?

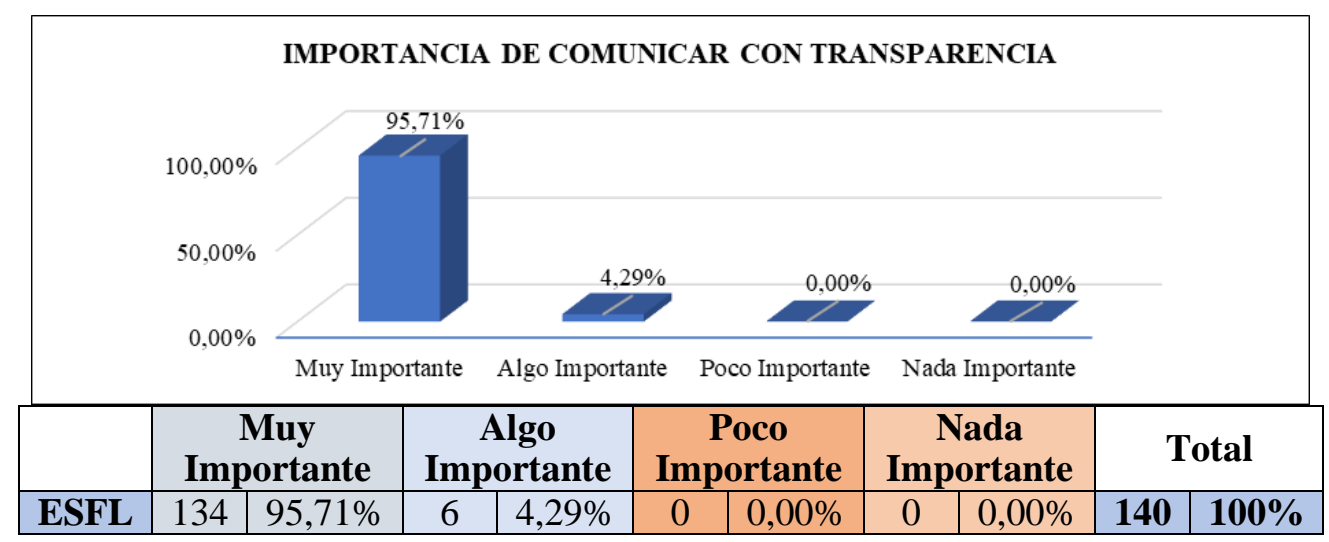

Fuente: Elaboración propia

\subsection{Conocimiento de los grupos de interés que deben ser considerados stakeholders de una ESFL}

Las actividades de una ESFL generan un interés en una diversidad de grupos que merecen ser considerados como stakeholders. Se va a tratar de determinar cuáles son los principales. 
Ilustración. 5. ¿Quiénes deben ser considerados los stakeholders de una ESFL?

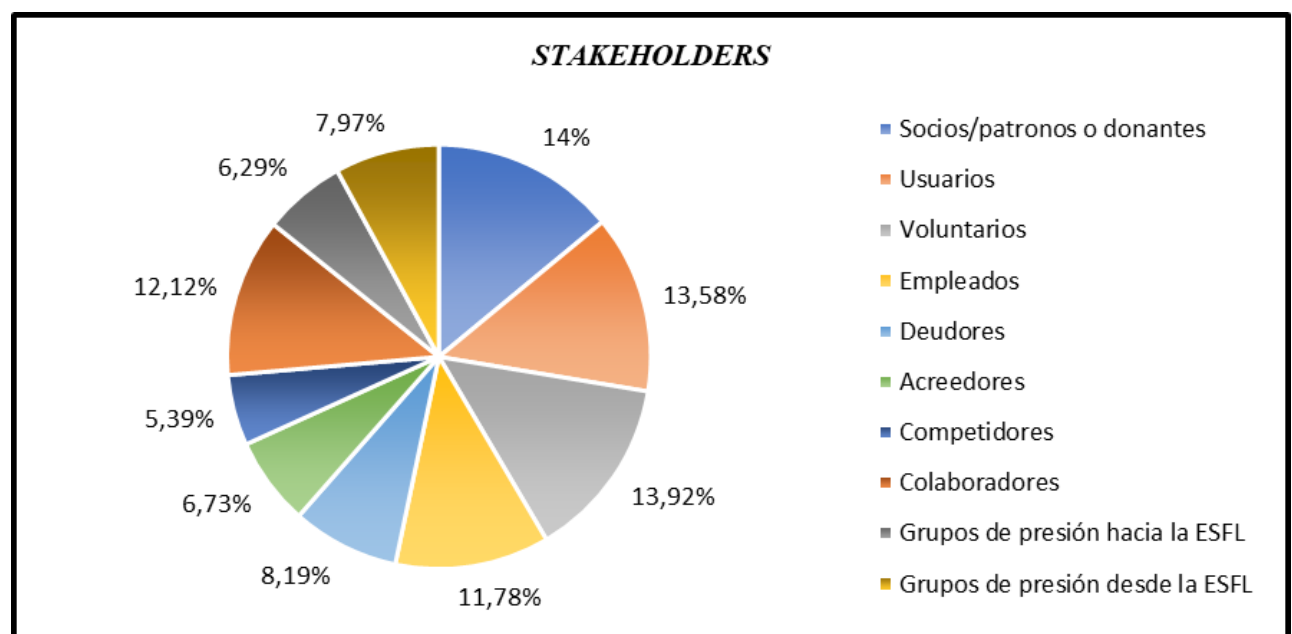

\begin{tabular}{|l|c|c|c|}
\hline \multirow{2}{*}{} & \multicolumn{3}{|c|}{ ESFL } \\
\cline { 2 - 4 } & $\begin{array}{c}\text { Total } \\
\text { Respuestas }\end{array}$ & $\%$ & \% s/Total \\
\hline Socios/patronos o donantes & 125 & $89.29 \%$ & $14.03 \%$ \\
\hline Usuarios & 121 & $86.43 \%$ & $13.58 \%$ \\
\hline Voluntarios & 124 & $88.57 \%$ & $13.92 \%$ \\
\hline Empleados & 105 & $75.00 \%$ & $11.78 \%$ \\
\hline Deudores & 73 & $52.14 \%$ & $8.19 \%$ \\
\hline Acreedores & 60 & $42.86 \%$ & $6.73 \%$ \\
\hline Competidores & 48 & $34.29 \%$ & $5.39 \%$ \\
\hline Colaboradores & 108 & $77.14 \%$ & $12.12 \%$ \\
\hline $\begin{array}{l}\text { Grupos de presión hacia la } \\
\text { ESFL }\end{array}$ & 56 & $40.00 \%$ & $6.29 \%$ \\
\hline $\begin{array}{l}\text { Grupos de presión desde la } \\
\text { ESFL }\end{array}$ & 71 & $50.71 \%$ & $7.97 \%$ \\
\hline \multicolumn{1}{|c|}{ Respuesta Múltiple } & 891 & & $100.00 \%$ \\
\hline
\end{tabular}

Fuente: Elaboración propia

Los socios, patronos, donantes y voluntarios son considerados como principales usuarios de la información que emiten las ESFL (ver Ilustración 5). Una vez determinado cuáles son los grupos de interés que deben ser considerados stakeholders de estas entidades, vamos a comprobar quiénes consideras las ESFL que deben demandar una información más transparente. 


\subsection{Identificación de los demandantes de una información más transparente a las ESFL}

No sólo es importante saber cuáles son los papeles o roles a desempeñar o qué tipo de stakeholder es cada agente, sino que se debe saber quiénes deben demandar una información transparente a las ESFL.

Ilustración. 6. ¿Quiénes deben demandar una información transparente a las ESFL?

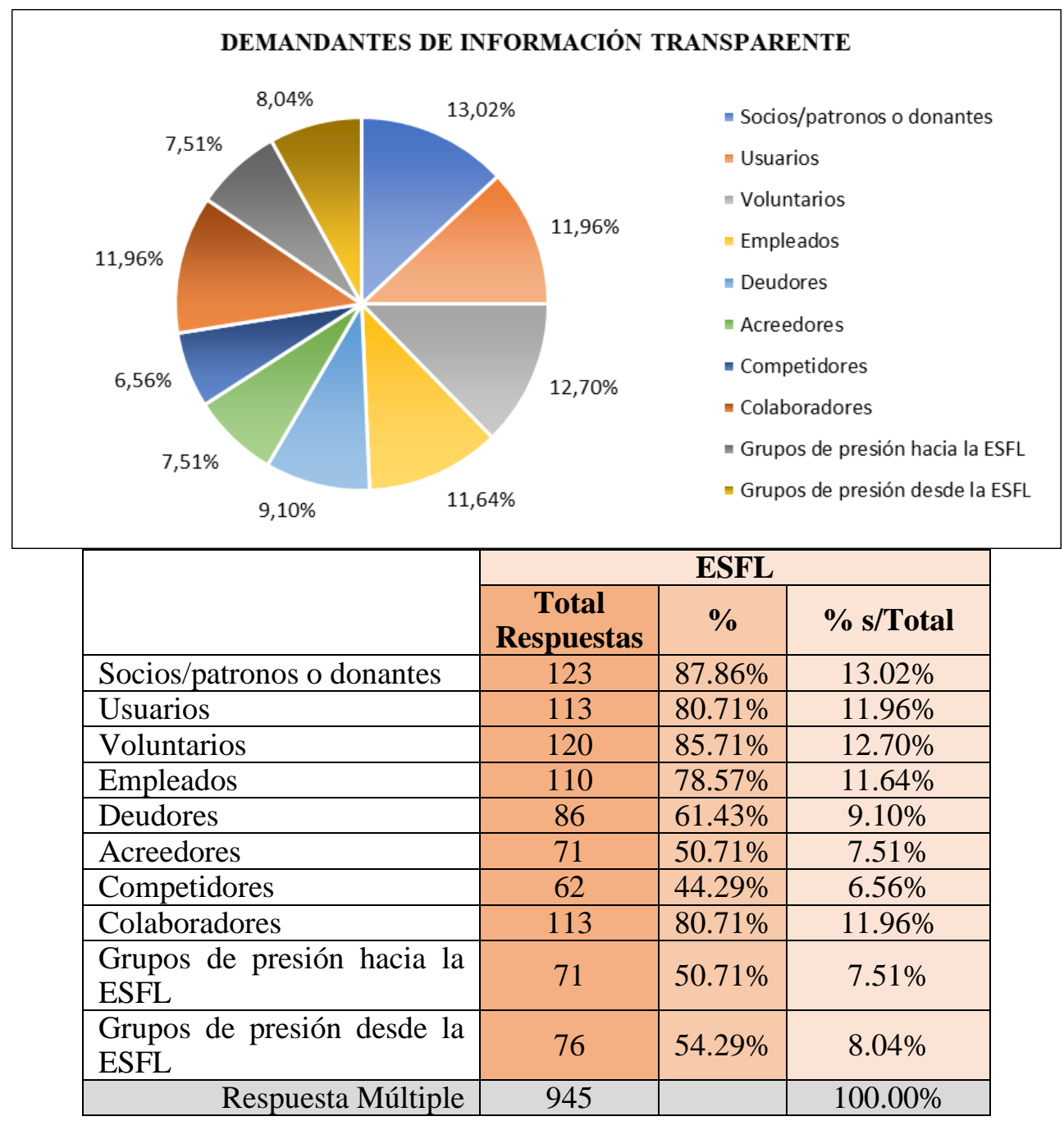

Fuente: Elaboración propia 
Los socios, patronos, donantes y voluntarios son los grupos de interés que las ESFL reconocen que deben demandar una información más transparente (ver Ilustración 6).

\subsection{Conocimiento de los atributos que aporta a las ESFL la emisión de información transparente}

Difundir una imagen positiva de cara a la sociedad y alcanzar el mayor compromiso social posible resulta imprescindible para que las ESFL obtengan los recursos económicos y humanos necesarios para el desarrollo de su actividad. Para ello, deben aprovechar cada oportunidad en mejoras continuas.

Ilustración. 7. ¿Qué atributos aporta a las ESFL ofrecer a sus stakeholders una información transparente?

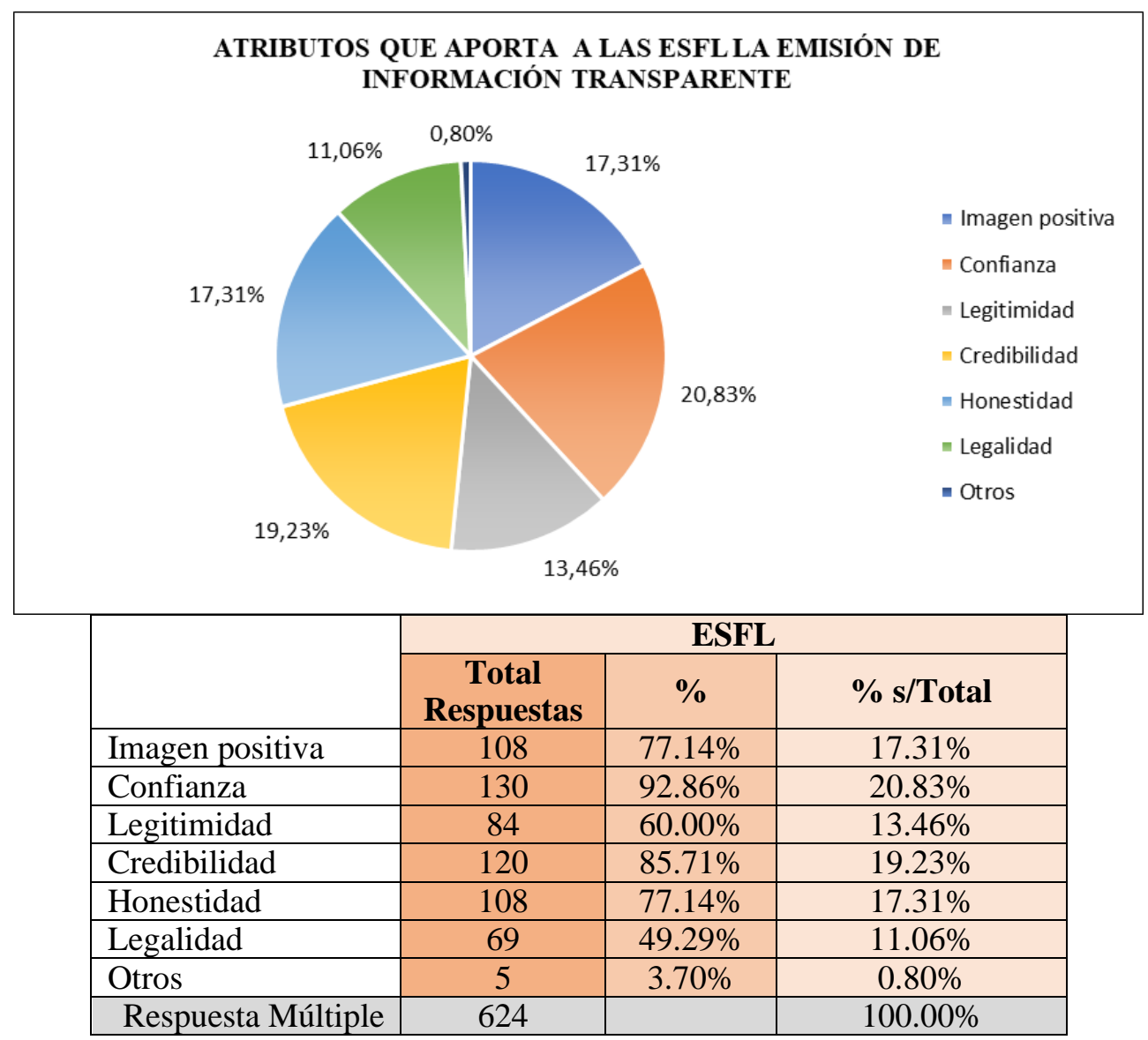

Fuente: Elaboración propia 
Confianza y credibilidad son los principales atributos que aporta a las ESFL el ofrecer a sus stakeholder una información transparente (ver Ilustración 7).

\subsection{Caracterización de la información emitida por las ESFL hacia sus stakeholders}

En la planificación estratégica de cualquier organización, la toma de decisiones es una actividad primordial. Para reducir el riesgo de que ésta se realice de manera incorrecta es preciso que la información emitida por las ESFL hacia sus grupos de interés cumpla una serie de requisitos.

Veracidad, claridad, fiabilidad y objetividad son las principales características que ha de tener la información emitida por las ESFL hacia sus grupos de interés. Sin dejar de lado el hecho de que dicha información sea también completa, útil y concisa (ver Ilustración 8).

Ilustración. 8. ¿Cómo ha de ser la información emitida por las ESFL?

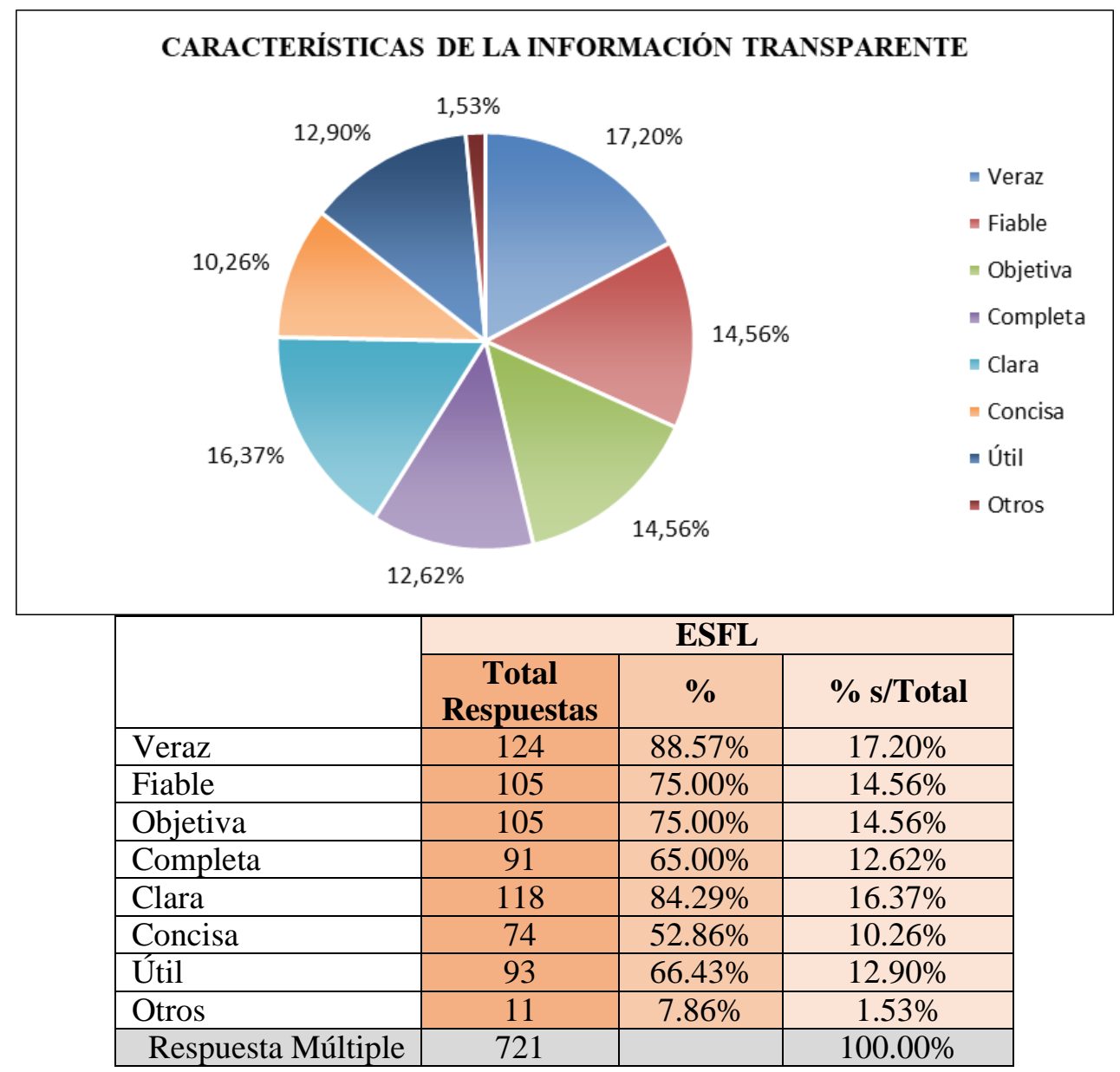

Fuente: Elaboración propia 


\section{Conclusiones}

Atendiendo a la revisión bibliográfica llevada a cabo y el estudio empírico desarrollado a través de tablas dinámicas, podemos concluir que:

Las ESFL son conscientes de la importancia de ofrecer una información transparente dirigida a sus grupos de interés. A pesar de ello, la normativa existente hasta el momento, a nivel nacional, no es muy amplia ni estricta en lo concerniente a qué, cómo y hacia quién ha de dirigirse la información. Por ello, aprovechando que las propias entidades son conocedoras de la relevancia de la transparencia, se hace esencial una normativa sustantiva propia que amplíe la publicación de mínimos sobre publicidad activa que realiza la vigente Ley de Transparencia, principalmente en lo referente a la información económica, presupuestaria, estadística y sobre todo social de las ESFL. Es decir, que tenga en cuenta todas las necesidades que demandan sus stakeholders en cuanto a emisión de información se refiere. Asimismo, resulta necesario que dicha normativa extienda los requisitos del ámbito de aplicación a aquellas ESFL que perciban también financiación de carácter privado.

A tenor de los resultados obtenidos, las ESFL perciben como grupos de interés, $\mathrm{y}$, por ende, con la potestad de demandar una información transparente, a cualquier persona física o jurídica que tenga algún tipo de relación directa o indirecta con ellas, siguiendo así la estela de la Teoría de los Stakeholders (Freeman, 1984). Sin embargo, no perciben como tales a los grupos de presión, tanto desde la propia entidad (lobbies) como hacia la propia organización, ni tampoco a otras entidades competidoras (entendidas éstas como aquellas que actúan en una actividad similar en un ámbito territorial determinado), circunstancia entendible si se tiene en cuenta la propia idiosincrasia de las ESFL. Éstas desarrollan su misión sin sentir la necesidad de competir contra nadie, por lo que no perciben a los competidores como un grupo de interés, porque simplemente, desde su punto de vista, no lo tienen.

Asimismo, cuando estas entidades actúan como grupos de presión frente a injusticias o vulneración de los derechos humanos, no se sienten como un grupo que genera presión en sí mismo, sino simplemente como una entidad que desarrolla su actividad, por lo que tampoco consideran a los grupos de presión como usuarios de su información.

Las ESFL, siguiendo los estudios de Herranz de la Casa (2006) y Balas-Lara (2011), proyectan una imagen positiva y logran visibilizar el Sector cuando comunican con transparencia. Además, así consiguen generar una mayor confianza y credibilidad social de la entidad. Por lo tanto, la emisión de información debe ser dirigida hacia sus grupos de interés mediante procesos de información y comunicación transparentes.

Además, la sociedad es cada vez más exigente y el rol que poseen en ella las ESFL establece un compromiso social. Por ello, tanto la emisión de información transparente de estas entidades hacia sus stakeholders como su comunicación, precisa de un proceso de educación y formación mediante dinámicas participativas donde se analice su situación real, se establezca un diagnóstico y se plantee cómo mejorarla. Esto no ha de ser considerado una carga adicional, sino un valor añadido para la organización. 
Como conclusión final señalar que la información emitida, según las propias ESFL, debe ser principalmente veraz, clara, fiable y objetiva. Para ello, resulta necesaria una normativa más detallada donde se marquen las pautas para ofrecer una información que cumpla, como mínimo, con esas características. Dicha información no debe ser sólo económica, sino también social y medioambiental.

\section{Futuras líneas de investigación}

Los stakeholders son agentes con una capacidad de influencia cada vez mayor, por lo que un proceso de identificación y clasificación es imprescindible para poder centrar los esfuerzos de las ESFL a la hora de conocer qué tipo de información demandan. Además, definir quiénes son y qué papeles desempeñan esos grupos de interés resulta una labor necesaria pero insuficiente para la misión de considerarlos como elementos creadores de ventaja competitiva para la organización, siendo preciso completar este primer paso con futuras investigaciones centradas en el análisis de sus intereses.

Por otra parte, es preciso indicar que la principal limitación del estudio radica en que los resultados son aplicables únicamente a las ESFL españolas. Por ello, una importante futura línea de investigación sería extrapolar el trabajo a otras regiones para identificar los stakeholders de las ESFL de esos países y examinar sus intereses.

\section{Referencias bibliográficas}

Álvarez, J. L. (2010) Corrupción a nivel nacional e internacional: La transparencia como antídoto. Implicaciones de la economía sumergida en España, pp. 149-172.

Álvarez-Etxeberria, I. (2009) Las memorias de sostenibilidad: un instrumento para la gestión de la sostenibilidad. Revista española de financiación y contabilidad, $\mathrm{N}^{\mathrm{o}} 38$, Vol. 144, pp. 677-697.

Balas-Lara, M. B. (2011) La gestión de la comunicación en el tercer sector: Análisis de la imagen percibida de las organizaciones del tercer sector.

Balas-Lara, M. B. (2008) El reto de la comunicación en el tercer sector no lucrativo. Revista Española del Tercer Sector, No 8, pp. 17-38.

Balser, D. y Mcclusky, J. (2005) Managing stakeholder relationships and nonprofit organization effectiveness. Nonprofit Management and Leadership, $\mathrm{N}^{\mathrm{o}}$ 15, Vol. 3, pp. 295-315.

Bebbington, J., Larrinaga, C. y Moneva, J. M. (2008) Corporate social reporting and reputation risk management. Accounting, Auditing y Accountability Journal, No 21 , Vol. 3, pp. 337-361.

Bernal, J. A. (2013) La información financiera de las entidades sin ánimo de lucro. Contaduría Universidad de Antioquia, № 55, pp. 149-164.

Brown, L. D. y Moore, M. H. (2001) Accountability, strategy and international nongovernmental organizations. Nonprofit and Voluntary Sector Quarterly, $\mathrm{N}^{\circ} 30$, Vol. 3, pp. 569-587. 
Caballero, G. (2006) Análisis de los stakeholders: Una reflexión sobre el poder de influencia en la organización. ICADE. Revista de Las Facultades de Derecho y Ciencias Económicas y Empresariales, $\mathrm{N}^{\circ}$ 68, pp. 101-122.

Callejo, J. (2002) Observación, entrevista y grupo de discusión: el silencio de tres prácticas de investigación. Revista Española de Salud Pública, No 76, Vol. 5, pp. 409-422.

Carrasco, F., Correa-Ruiz, C. y Larrinaga, C. (1999) Información y gestión medioambiental: una reflexión sobre los factores de motivación, proceso de desarrollo de informes e influencia de los stakeholders en las empresas del sector eléctrico.

Cavanna, J. (2013) Construir confianza 2012. Impulsando la transparencia en la web de las fundaciones españolas. Fundación Compromiso y Transparencia. Consultado el 2 de abril de 2013, desde http://http://spainsif.es/sites/default/files/upload/publicaciones/Construirconfianza2013. pdf.

Clarkson, M. E. (1995) A stakeholder framework for analyzing and evaluating corporate social performance. Academy of Management Review, $\mathrm{N}^{\circ}$ 20, Vol. 1, pp. 92-117.

Donaldson, T. y Preston, L. E. (1995) The stakeholder theory of the corporation. The Academy of Management Review, No 20, Vol. 1, pp. 65-91.

Elechiguerra, C., Corral-Lage, J. y Maguregui, M. L. (2015) La Gestión de Asociaciones y Fundaciones: Calidad Y Transparencia. Madrid: Pirámide.

Escobar, B. y González, J. M. (2005) Responsabilidad social corporativa: ¿compromiso u oportunismo? Revista de Contabilidad, No8, Vol. 16, pp. 67-98.

Fisher, K. L. (2015) Swings for Dreams: Public Perceptions of the Nonprofit Sector and Effects on Donating Behavior.

Fottler, M. D. (1987) Health care organizational performance: Present and future research. Journal of Management, $\mathrm{N}^{\circ} 13$, Vol. 2, pp. 367-391.

Freeman, R. E. (1984) Strategic management: A stakeholder approach. Advances in strategic management, $\mathrm{N}^{\mathrm{o}} 1$, Vol. 1, pp. 31-60.

Friedman, M. (1962) Price theory: A provisional text. Chicago: Aldine.

Gabas, F., Moneva, J. M., Bellostas, A. J. y Jarne, J. I. (1996) Análisis de la demanda de información financiera en la coyuntura actual. Revista española de financiación y contabilidad, $\mathrm{N}^{\mathrm{o}}$ 86, pp. 103-137.

García-Gutiérrez Fernández, C. (2002) La empresa de participación: características que la definen. Virtualidad y perspectivas en la sociedad de la información. CIRIEC-España, Revista de Economía Pública, Social y Cooperativa, No 40, pp. 99-122.

Goodpaster, K. E. (1991) Business ethics and stakeholder analysis. Business Ethics Quarterly, pp. 53-73.

Gray, R., Dey, C., Owen, D., Evans, R. y Zadek, S. (1997) Struggling with the praxis of social accounting: stakeholders, accountability, audits and procedures. Accounting, Auditing y Accountability Journal, № 10, Vol. 3, pp. 325-364.

Gruber, J. E. (1987) Controlling bureaucracies: Dilemmas in democratic governance. University of California Print.

Grunig, J. E. y Hunt, T. (1984) Managing public relations (21-23). New York: Holt, Rinehart and Winston.

Herman, R. D. y Renz, D. O. (1999) Theses on nonprofit organizational effectiveness. Nonprofit and Voluntary Sector Quarterly, No 28, Vol. 2, pp. 107-126.

Herranz De La Casa, J. M. (2006) La comunicación y la transparencia en las organizaciones no lucrativas. Universidad Complutense de Madrid, Servicio de Publicaciones.

Herlin, H. (2015) Better safe than sorry: Nonprofit organizational legitimacy and crosssector partnerships. Business y Society, № 54, Vol. 6, pp. 822-858. 
Horrach, P. y Socías-Salvà, A. (2011) La actitud de las empresas de economía solidaria frente a la divulgación de información sobre sostenibilidad desde el prisma de la teoría de los stakeholders o grupos de interés. Revista de Contabilidad, No 14, pp. 267-297.

Huang, C. C. (2017) The Development of China's Nonprofit Sector since 1995. In China's Nonprofit Sector, pp. 21-38. Routledge.

Johnson, G., Scholes, K. y Fréry, F. (2000) Stratégique. Publi-Union.

Kaler, J. (2002) Morality and strategy in stakeholder identification. Journal of Business Ethics, № 39, Vol. 1-2, pp. 91-100.

Kearns, K. P. (1994) The strategic management of accountability in nonprofit organizations: An analytical framework. Public Administration Review, pp. 185-192.

Kearns, K. P. (2001) Transparencia en las ONG: Un enfoque proactivo. La transparencia de la solidaridad, $\mathrm{N}^{\circ}$ 1, pp. 51-66. Comunidad de Madrid: Fundación Lealtad.

Larrinaga, C., Carrasco, F., Correa-Ruíz, C., Llena, F. y Moneva, J. M. (2002) Accountability and accounting regulation: the case of the Spanish environmental disclosure standard. European Accounting Review, № 11, Vol. 4, pp. 723-740.

Marcuello, C., Bellostas, A. J., Marcuello, C. y Moneva, J. M. (2007) Transparencia y rendición de cuentas en las empresas de inserción. CIRIEC-España. Revista de Economía Pública, Social y Cooperativa, № 59, pp. 91-122.

Mitchell, R. K., Agle, B. R. y Wood, D. J. (1997) Toward a theory of stakeholder identification and salience: Defining the principle of who and what really counts. Academy of Management Review, $\mathrm{N}^{\circ} 22$, Vol. 4, pp. 853-886.

Moneva, J. y Bellostas, A. (2007) Accountability en las entidades sin fines lucrativos. Centros De Investigación de Economía y Sociedad.

Montero, M. J. y Araque, R. (2006) Análisis de los stakeholders en una ENL. Dirección de entidades no lucrativas: Marco jurídico, análisis estratégico y gestión. Editorial Aranzadi.

Observatorio del Tercer Sector (OTS) (2007) Transparencia y rendición de cuentas en las organizaciones no lucrativas. Retrieved from http://www.tercer-sector.org/.

Peng, S., Pandey, S. y Pandey, S. K. (2015) Is there a nonprofit advantage? Examining the impact of institutional context on individual-organizational value congruence. Public Administration Review, № 75, Vol. 4, pp. 585-596.

Pérez Fernández, E. (2002) La participación como elemento constitutivo de las empresas de la Nueva Economía Social. CIRIEC-España, Revista de Economía Pública, Social y Cooperativa, No 40, pp. 141-163.

Post, J. E., Preston, L. E. y Sachs, S. (2002) Redefining the corporation: Stakeholder management and organizational wealth. Stanford University Press.

Rhine, A. S. (2015) An examination of the perceptions of stakeholders on authentic leadership in strategic planning in nonprofit arts organizations. The Journal of Arts Management, Law, and Society, $\mathrm{N}^{\circ}$ 45. Vol. 1, pp. 3-21.

Salamon, L. M. y Sokolowski, S. W. (2016) Beyond nonprofits: Re-conceptualizing the third sector. Voluntas-International Journal of Voluntary and Nonprofit Organizations, No 27, Vol. 4, pp. 1515-1545.

Salvador I Peris, S. (2003) Comunicación en imagen en las ONG. Jornades de Foment de la Investigació, Universitat Jaume I Castellón.

Saz-Gil, M. (2008) Introducción a la gestión Stakeholder en las organizaciones no lucrativas. Una aproximación a través del estudio de casos. Estudios de Economía Aplicada, $\mathrm{N}^{\mathrm{o}} 26$, Vol. 1.

Shafritz, J. M. (1992) The HarperCollins dictionary of American Government and politics Harper Perennial. 
Starik, M. (1994) The Toronto conference: Reflections on stakeholder theory. Business $y$ Society, No 33, Vol 1, pp. 82-131.

Starling, G. (2010) Managing the public sector Wadsworth Pub Co.

Steadman, M. E., Zimmerer, T. W. y Green, R. F. (1995) Pressures from stakeholders hit Japanese companies. Long Range Planning, $\mathrm{N}^{\circ} 28$, Vol. 6, pp. 29-37.

Thompson, J. K., Wartick, S. L. y Smith, H. L. (1991) Integrating corporate social performance and stakeholder management: Implications for a research agenda in small business. Research in corporate social performance and policy, $\mathrm{N}^{\circ} 12$, Vol. 1, pp. 207230.

Unerman, J. (2007) Stakeholder engagement and dialogue. Sustainability accounting and accountability, $\mathrm{N}^{\mathrm{o}} 86$.

Unerman, J. y Bennett, M. (2004) Increased stakeholder dialogue and the internet: towards greater corporate accountability or reinforcing capitalist hegemony? Accounting, Organizations and Society, $\mathrm{N}^{\mathrm{o}} 29$, Vol. 7, pp. 685-707.

Val, M. R., Bernat, F. J. y Johanisova, N. (2012) Transparencia y rendición de cuentas en las Entidades No Lucrativas. El caso de las fundaciones. Revista de Contabilidad y Dirección, № 14, pp. 159-181.

Vaccaro, A. y Madsen, P. (2009) ICT and an NGO: Difficulties in attempting to be extremely transparent. Ethics and Information Technology, № 11, Vol. 3, pp. 221-231.

Vidal, P., Grabulosa, L. y Observatorio del Tercer Sector. (2008) Qué es la transparencia en una ONG. Alzheimer de la Asociación Afal Contigo, No 51, pp. 38-39.

Waters, R. D., Burnett, E., Lamm, A. y Lucas, J. (2009) Engaging stakeholders through social networking: How nonprofit organizations are using Facebook. Public Relations Review, № 35, Vol. 2, pp. 102-106.

Zhang, X., Griffith, J., Pershing, J., Sun, J., Malakoff, L., Marsland, W. y Field, E. (2017) Strengthening Organizational Capacity and Practices for High-Performing Nonprofit Organizations: Evidence from the National Assessment of the Social Innovation FundA Public-Private Partnership. Public Administration Quarterly, № 41, Vol. 3. 